\title{
Sleep Analysis for Elderly Care using a Low-resolution Visual Sensor Network
}

\author{
Mohamed Eldib ${ }^{1}$, Francis Deboeverie ${ }^{1}$ \\ Wilfried Philips ${ }^{1}$, and Hamid Aghajan ${ }^{1,2}$
}

TELIN-IPI-IMINDS, Ghent University, Sint-Pietersnieuwstraat 41, Ghent, Belgium Department of Electrical Engineering, 350 Serra Mall, Stanford University, CA, USA

\begin{abstract}
Nearly half of the senior citizens report difficulty initiating and maintaining sleep. Frequent visits to the bathroom in the middle of the night is considered as one of the major reasons for sleep disorder. This leads to serious diseases such as depression and diabetes. In this paper, we propose to use a network of cheap low-resolution visual sensors $(30 \times 30$ pixels $)$ for long-term activity analysis of a senior citizen in a service flat. The main focus of our research is on elderly behaviour analysis to detect health deterioration. Specifically, this paper treats the analysis of sleep patterns. Firstly, motion patterns are detected. Then, a rule-based approach on the motion patterns is proposed to determine the wake up time and sleep time. The nightly bathroom visit is identified using a classification-based model. In our evaluation, we performed experiments on 10 months of real-life data. The ground truth is collected from the diaries in which the senior citizen wrote down his sleep time and wake up time. The results show accurate extraction of the sleep durations with an overall Mean Absolute Error (MAE) of 22.91 minutes and Spearman correlation coefficient of 0.69. Finally, the nightly bathroom visits analysis indicate sleep disorder in several nights.
\end{abstract}

Keywords: Sleep disorder, sleep analysis, visual sensor networks, ambient assisted living

\section{Introduction}

Sleeping is of the utmost importance to everyone, especially for the elderly. According to the following study [8], the authors have found that $30 \%$ of the people have trouble to sleep. In another study [7] on more than 9000 elderly subjects, more than 4500 subjects have reported difficulty falling asleep due to frequent visits to the bathroom at night for voiding, waking up too early, or sleeping late.

Nocturia is defined as the awakening from sleep to urinate after an individual has gone to sleep. In a recent study [20], Nocturia-related awakenings are reported to cause significant sleep disorder and fatigue in elderly patients. Sleep disorder increases the risk of poor physical function and decreases the cognitive function. This dangers the quality of life of the senior citizens. The disease associated with insufficient sleep for the senior citizens have a significant impact on 
health care. In the approaching decade, the institutions that take care of the senior citizens will run into operational and financial problems in the approaching decade.

Aging-in-place presents itself as a promising solution for health care systems. Recently, aging-in-place has gained a lot of attention in recent years due to the fact that many senior citizens prefer to age and maintain their independence as long as possible in their own homes [5] because of the emotional and physical associations, memories and comfort. Aging-in-place promotes well-being of older people without scarifying the quality of life in a familiar environment and maintains the valuable social networks. The success of aging-in-place depends on Ambient Assisted Living (AAL) tools which have witnessed tremendous improvements in the last few years. AAL tools provide supervision and assistance with activities of daily living (ADL) to prevent, cure, and improve wellness and health conditions of seniors.

We have been monitoring a senior citizen living in a service flat in Belgium for one year using a network of 10 low-resolution visual sensors. In this paper, we discuss our efforts to analyze sleep patterns. We are still developing approaches and algorithms to recognize more ADLs to detect increasing or decreasing health conditions. The main contributions of this paper are: (1) indirect detection of sleep durations and nightly bathroom visits. (2) The evaluation of the sleep analysis approach on a real-life dataset covering 10 months by comparing the results to ground truth, and (3) studying the relation between sleep disorder and the number of the nightly bathroom visits.

The paper is organized as follows. In section 2 , we present related work. Section 3 explains our approach of detecting sleep durations and nightly bathroom visits. Section 4 describes our experimental results. Finally, Section 5 offers some conclusions.

\section{Related Work}

The sensors used in AAL tools can be divided into two main categories: (1) wearable sensors, smartphone and (2) ambient sensors. In the first category $[10,2]$, various wearable sensors, such as accelerometers, gyroscopes, proximity sensors and e-textile sensors, could be attached to the subject of interest to monitor vital signs such the heart beats, the respiration, the blood pressure and the glucose level and the muscle activity. In [16] the authors used a chest strap and a wristband wearable devices to analyze the sleep patterns, and to identify the most relevant sleep parameters for stress detection. They evaluated their approach on 10 healthy pilgrims for 18 days. Zhenyu Chen et al. [4] presented an approach for measuring sleep duration based on a novel best effort sleep (BES) model. The BES model infers sleep using smartphones in a completely unobtrusive way. Their approach achieved an accuracy of 42 minute on 8-persons for 7 days. Bed occupancy sensor (BOS), tele-actimeter and sleep logs have been used in [14] to analyze total sleep time and sleep quality. However, wearable sensors and smartphone face a few disadvantages such as battery life, high cost, 
being forgotten to be worn by the user, and the requirement to be worn on specific body parts to provide reliable measurements.

In the second category, ambient sensors are installed in the home environment by mounting them on the wall or the ceiling, embedded in furnitures and appliances. Passive Infrared Motion (PIR) sensors, visual sensors and Radio Frequency Identification (RFID) are the most popular among researchers. In [17], the authors used pressure sensor matrix to monitor the elders sleep behavior in bed. Enamul Hoque et al. [11] presented a sleep monitoring system based on active RFID-based sensors equipped with accelerometers. They evaluated their system for six nights on one subject. Despite the popularity of PIR sensors, they are known of having the following problems: (1) highly bursty output which limits PIR systems to single-person scenarios; (2) self-triggering due to sudden changes in environmental conditions such as heating, ventilating, and air conditioning; (3) PIR cannot sense people who are standing still [19].

There has been a few attempts to use camera for sleep analysis. In [9], the authors proposed a camera-based system combining video motion detection, motion estimation, and texture analysis with machine learning for sleep analysis. Their system should be installed in front of the bed to perform the sleep analysis. They used six video sequences with six different subjects to validate their system. A sleep monitoring system using Kinect has been proposed in [13]. The system used depth and infrared images to track the body for posture and movements analysis. The system has been evaluated on 20 simulated use-cases for 7 hours. In [15]. the authors used Kinect and contact-based pressure mattress to build a sleep monitoring system. The system combined rule-based and supervised learning methods to recognize sleep patterns of 7 different individuals simulating their sleep habits. In another study [12], the authors introduced a Kinect-base setup where a number of measures are computed to quantify the amount of relevant motion. Then, a binary classifier is used for the actual sleep detection.

Our approach of sleep analysis is different than the work in $[9,13,15]$. The authors used a high-resolution camera in front of the bed for sleep analysis which raises concerns about the privacy. In contrast, we opted to use low-resolution cameras, and not cameras with regular imaging resolutions which often raise privacy concerns and increase the cost of the sensor network. Also, no visual sensor has been installed inside the bedroom for sleep analysis. In $[16,4,9,11]$, they performed sleep analysis on small datasets (several days) and the datasets were captured in lab environments. On the contrary, we perform long-term sleep analysis on 10 months of real-data recordings in a real service flat setup.

\section{Sleep Analysis Approach}

We used the low-resolution visual sensors [3] in previous studies $[6,18]$ for person tracking in our lab, as a first step, towards building a sensor network in a service flat for activity analysis. The sensor network deployed in the service flat is composed of 10 cameras as shown in Figure 1. Each camera includes a stereo 
pair of visual sensors producing images of $30 \times 30$ pixels, and a digital signal controller. The visual sensor images often suffer from artifacts due to read out problems such as electrical interference, and it does not have built-in processing capabilities such as lens shading correction resulting in a reduction of the image's brightness (vignetting). This can be solved by performing devignetting on the digital signal controller.
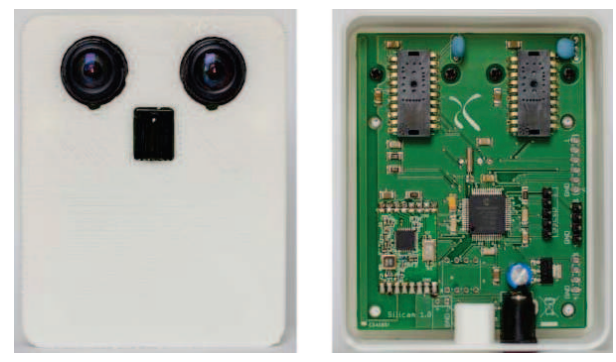

Fig. 1. The camera consists of stereo pair of image sensors controlled by a digital signal controller. Each image sensor delivers an image with a resolution of $30 \times 30$ pixels.

The results in this paper are obtained from a system setup in a service flat, covering an area of $8 \times 4.4 \mathrm{~m}^{2}$. Figure 2 displays the living space layout with camera positions. The voluntary older person participating in the installation is 83 years old with diabetes and decreased mobility due to a partial paralysis. However, the resident has a very clear mind. No instructions were given to the senior citizen about the way and the time of performing the sleep activity. The senior citizen was hospitalized in April 2014, December 2014 and January 2015 for 18,8 and 9 days respectively.

One of the major challenges of the current setup is the unavailability of the visual sensors in the bedroom and the bathroom which increase the difficulty of detecting accurate sleep durations and nightly bathroom visits.

We propose a simple approach that estimates the presence of the senior citizen in the room. Let $p_{k}(t)$ be the average number of foreground pixels in frames $t \ldots t+L-1$ of camera $k$. Then, we output a motion feature vector $\vec{x}_{t}=\left(p_{k}(t) \ldots p_{m}(t)\right)$ where $m$ is the number of the visual sensors. In this paper, we opted to use the correlation method as a foreground/background subtraction algorithm for its good performance with the visual sensor of [3], as indicated in a previous study [18]. The obtained results were good enough for detecting motion patterns produced by the senior citizen as shown in Figure 3.

We use a rule-based approach to detect the wake up time and sleep time. The inference rules have the generic form: IF A AND/OR B THEN C. The wake up time is detected when the senior citizen produces sufficient motion in the service flat in the morning. The motion should last for several minutes (e.g., more than 30 minutes) to indicate the senior citizen has actually woken up, and not to be 


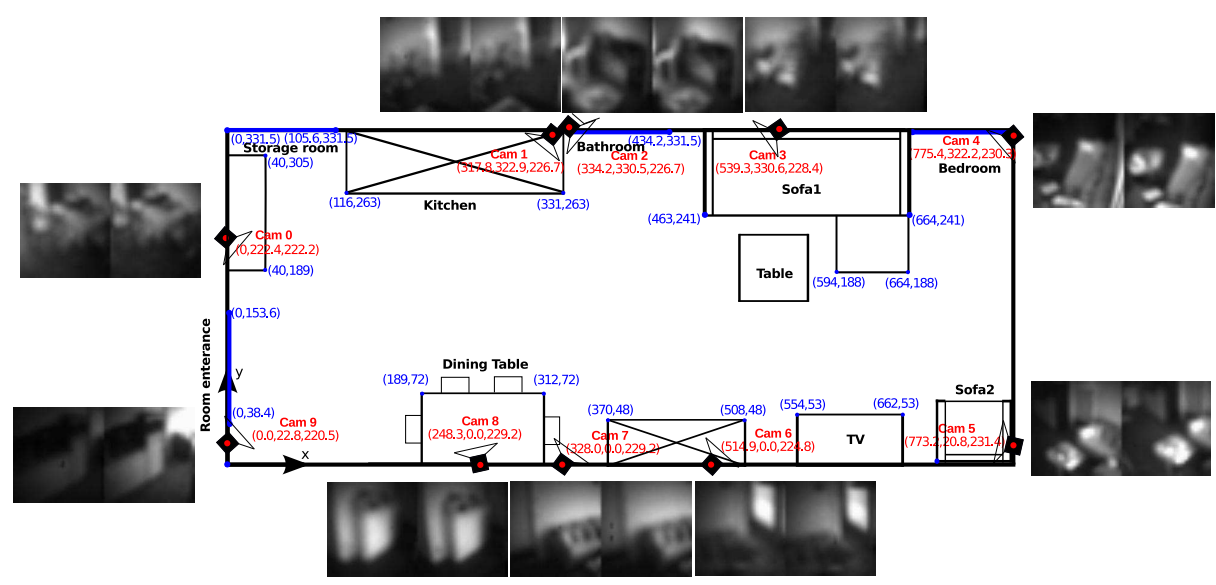

Fig. 2. Living space layout showing the configuration of ten visual sensors (each containing stereo image sensor) covering an area of $8 \times 4.4 \mathrm{~m}^{2}$.

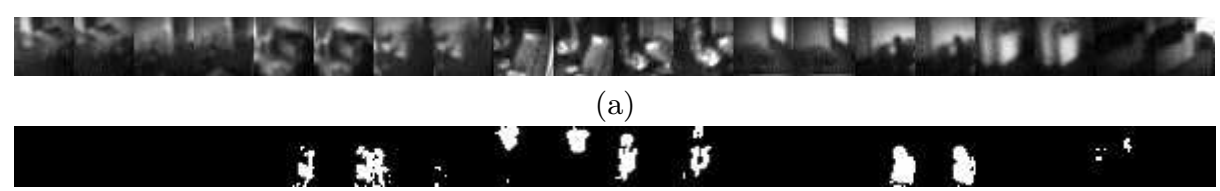

(b)

Fig. 3. (a) Original images, (b) foreground images produced by the correlation method.

confused with the nightly bathroom visit. Wherein, sleep time is detected by considering the last motion the senior citizen produces (e.g., turning off the TV at night). The TV status is detected by computing the intensity values of the TV's region of interest. If the intensity proceeds a threshold, then the TV status is on. Otherwise, the TV status is off.

Let $w_{d}$ be the wake up time of day $d$ and $s_{d-1}$ be the sleep time of day $d-1$. Then, the sleep duration $u_{d}$ of day $d$ is computed as

$$
u_{d}=w_{d}-s_{d-1}
$$

For the nightly bathroom visits, we use a Support Vector Machine (SVM) classifier with RBF kernel for detecting the nightly bathroom visit. The training data of the nightly bathroom visit $D$ is defined as:

$$
D=\left\{\vec{x}_{i}, y_{i} \mid y_{i} \in\{0,1\}\right\}_{i=1}^{n},
$$

where the $y_{i}$ is either 0 (not in the bathroom) or 1 (inside the bathroom), indicating the the class to which the feature vector $\vec{x}_{i}$ belongs. In the training 
step, We annotate one week of data containing the nightly bathroom visits. Then, we use the annotated data to build a SVM model. After building the model, the motion feature vectors are computed for the test days and classified. Finally, the nightly bathroom visit is valid when:

1. The bathroom visit happens after 30 minutes from the time the senior citizen goes to sleep.

2. There is a time difference of 60 minutes between two consecutive bathroom visits.

3. The bathroom visits take place between the bedtime and $5 \mathrm{am}$.

\section{Experiments}

To validate the performance of our proposed approach, we collected 10 months of real-life recordings using a network of 10 low-resolution visual sensors producing an image of $30 \times 30$ pixels at a frame rate of $50 \mathrm{fps}$. Video capturing is timesynchronized. The data of each day corresponds to a 24 hour period starting from midnight.

In this section, we compare the estimated sleep durations against ground truth. The ground truth is collected from diaries in which the elderly wrote down his sleep time and wake up time. The ground truth obtained from the diary has been validated by an informal caregiver (e.g. family member). The diaries are noted everyday, except for a few weeks when the caregiver forgot to give the senior citizen new diaries. The data interpreted has been demonstrated to some caregivers in the project meetings and workshops. The caregivers were excited about what is possible from the visual sensor data analysis.

Figure 4 shows the comparison between the estimates and the ground truth of sleep duration for two different periods. The vertical error bars in Figure 4 shows the overestimates and the underestimates of sleep durations. About $20 \%$ of the cases are overestimated and $6 \%$ of the cases are underestimated by more than 30 minutes. There are more overestimates than underestimates of sleep durations due to standing up without putting the lights on. Figure 5 shows the estimates and the ground truth of the wake up time. The overestimates of the wake up time can be clearly seen in the winter period (Figure 5b) than the summer period (Figure 5b). Similarly, Figure 6 shows the estimates and the ground truth of the sleep time.

Next, we compute the cumulative score (CS) to evaluate the sleep duration performance within error ranges. The cumulative score $C S(j)$ is defined as $\frac{N_{e \leq j}}{N} \times 100 \%$ where $N_{e \leq j}$ is the number of days on which sleep duration estimates have an absolute error not higher than $j$ minutes. Figure 7 shows the CS of the sleep duration estimates. At an absolute error range of 25 minutes, we achieve an accuracy of $70 \%$. The accuracy reaches $80 \%$ with an absolute error range of 40 minutes. We use the Mean Absolute Error (MAE) to measure the performance of the senior citizen sleep duration estimates: 


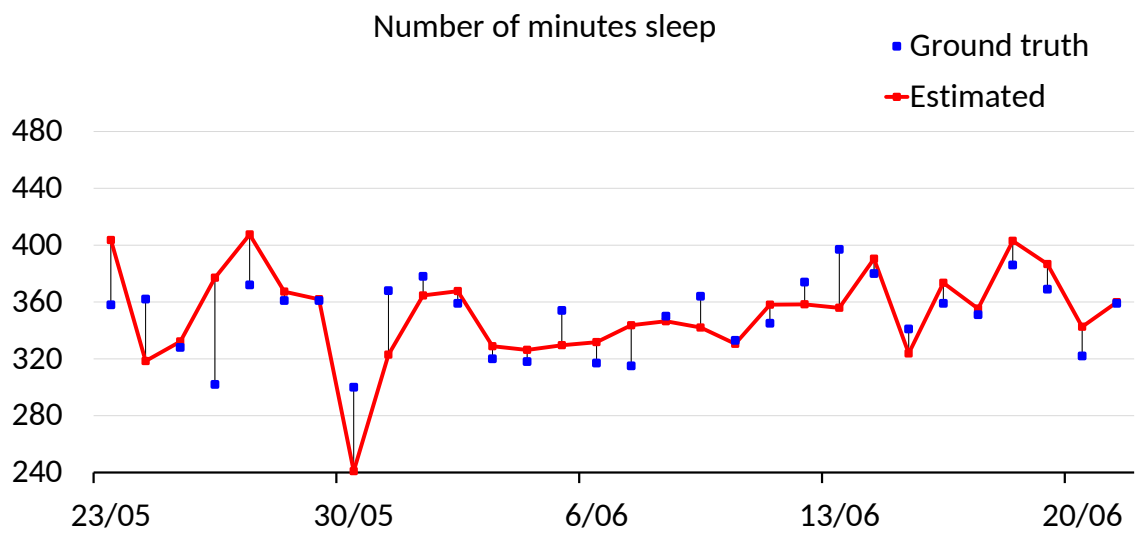

(a)

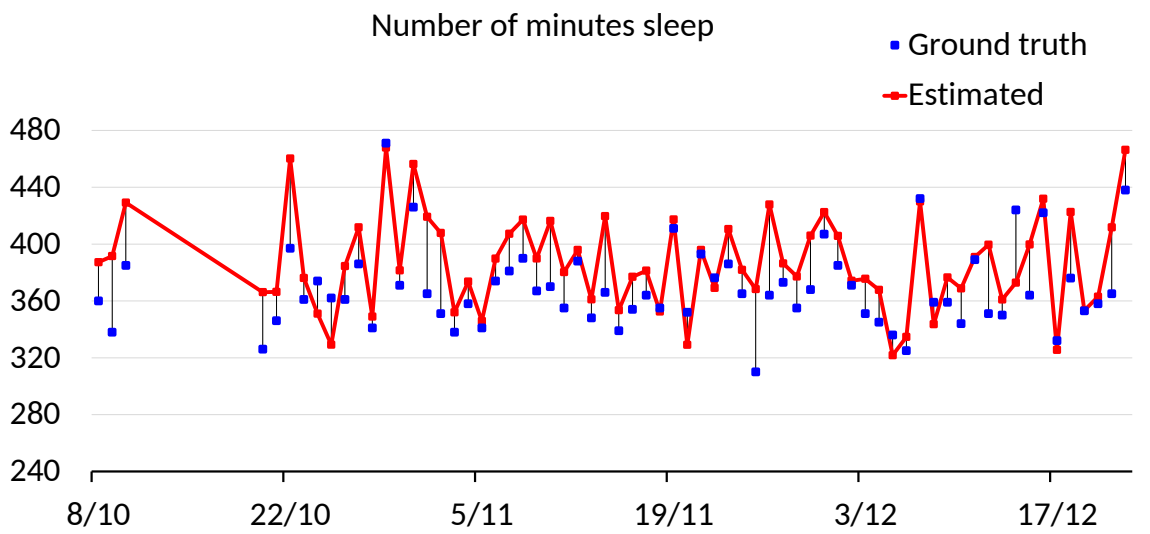

(b)

Fig. 4. A comparison between estimates and ground truth of sleep duration. The vertical error bars show the overestimates and the underestimates of sleep duration: (a) May and June, (b) October, November and December.

$$
M A E=\sum_{d=1}^{N} \frac{\left|u_{d}-u_{d}^{\prime}\right|}{N},
$$

where $u_{d}$ is the sleep duration estimate of day $d, u_{d}^{\prime}$ is the actual sleep duration of day $d$ and $N$ is the number of ground truth days. the MAE of the sleep duration estimates is 22.91 minutes. Then, The Relative Absolute Error (RAE) is computed to measure the error percent: 


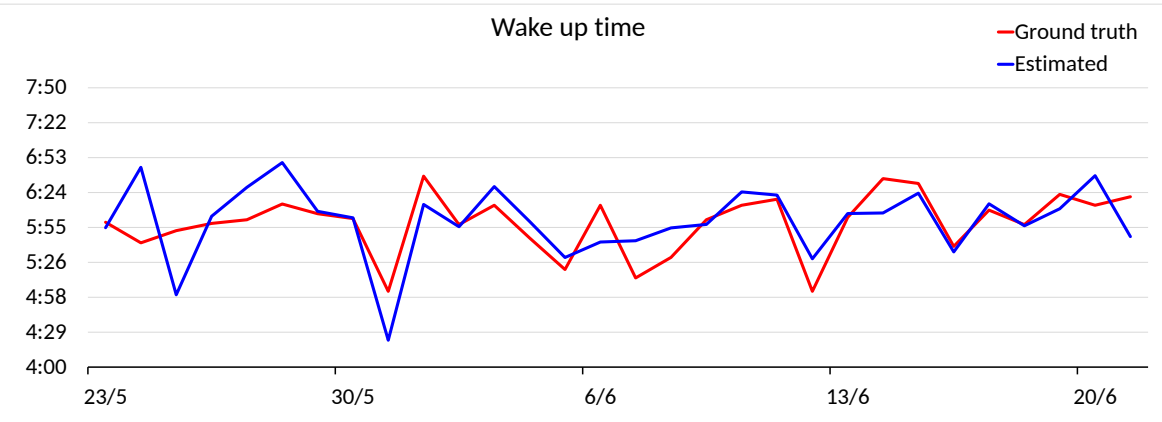

(a)

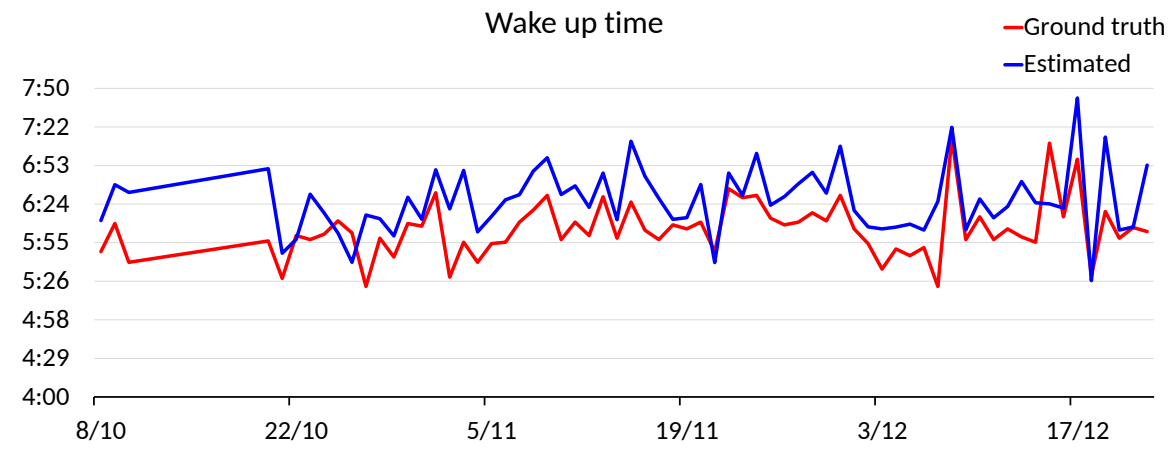

(b)

Fig. 5. A comparison between the estimates and the ground truth of wake up time: (a) May and June, (b) October, November and December.

$$
R A E=\frac{\sum_{d=1}^{N} \frac{\left|u_{d}-u_{d}^{\prime}\right|}{u_{d}^{\prime}}}{N} \times 100
$$

The RAE of the sleep duration is $6.39 \%$. Despite waking up without putting the lights on, our approach of estimating the sleep duration provides promising results close to the ground truth. The accuracy can be increased by using other sensors inside the bed room such as PIR sensors and thermopiles.

Finally, we measure the Spearman's rank correlation coefficient to assess how well the relationship between the sleep duration estimates and the ground truth. Let $u_{i}$ be the sleep duration estimates set, and let $\pi_{u_{i}}$ be the set of corresponding ranks. Similarly, let $\pi_{u_{i}^{\prime}}$ be the ranks of the ground truth set $u^{\prime} i$. Let $d_{i}$ be the difference between the two ranks $\pi_{u_{i}}$ and $\pi_{u_{i}}$. The Spearman's rank correlation coefficient $r_{s}$ is defined as:

$$
r_{s}=1-\frac{6 d_{i}^{2}}{n\left(n^{2}-1\right)}
$$




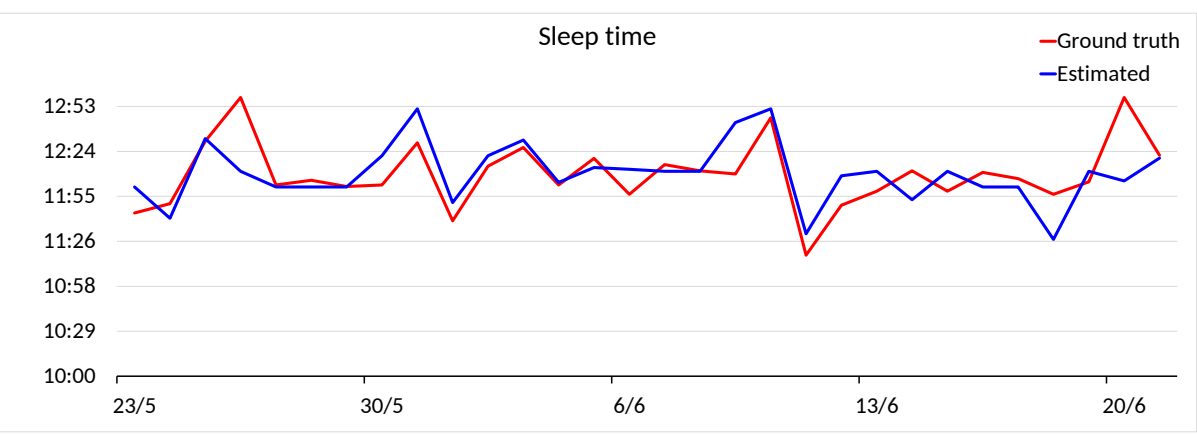

(a)

Sleep time

-Ground truth -Estimated

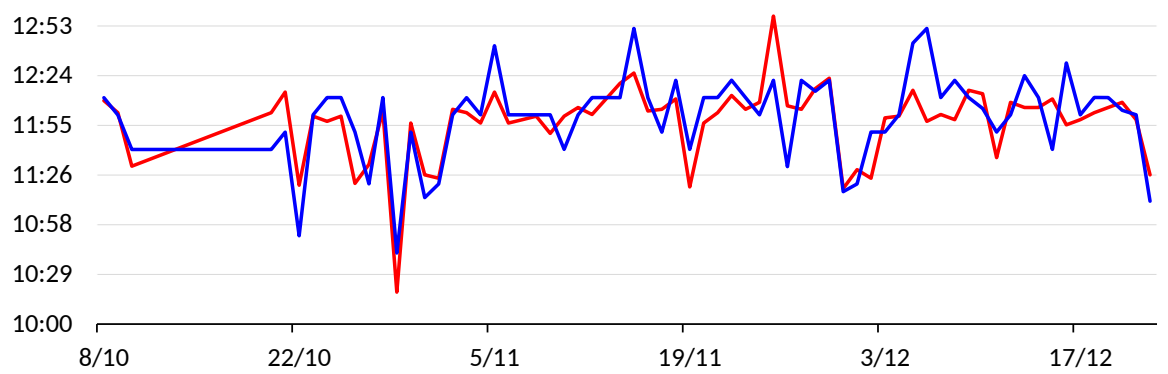

(b)

Fig. 6. A comparison between the estimates and the ground truth of sleep time: (a) May and June, (b) October, November and December.

where $n$ is the number of ground truth days. The Spearman correlation coefficient $r_{s}$ is 0.69 . This value shows that the correlation between the sleep duration estimates and the ground truth is high. Figure 8 shows the correlation between the sleep duration estimates and the ground truth.

Next, we measure the number of the bathroom visits in the middle of the night to discover sleep disorders. We used SVM for detecting the nightly bathroom visits. $10 \%$ of the output results (30 days) from the SVM have been visually checked. In most of the cases, the nightly bathroom visits were detected correctly. In few cases, the nightly bathroom visits were not detected due to standing up without putting the lights on. Figure 9 shows the number of the nightly bathroom visits per day from April to December. The senior citizen goes to the bathroom on average from one to two times per day. There are few days when the senior citizen went to the bathroom for three times per day. These days are possible indication of sleep disorder [1]. Figure 10 shows the number of the nightly bathroom visits per month. There are more nightly bathroom visits in June and August (46) than in the other months. This indicates a high possi- 


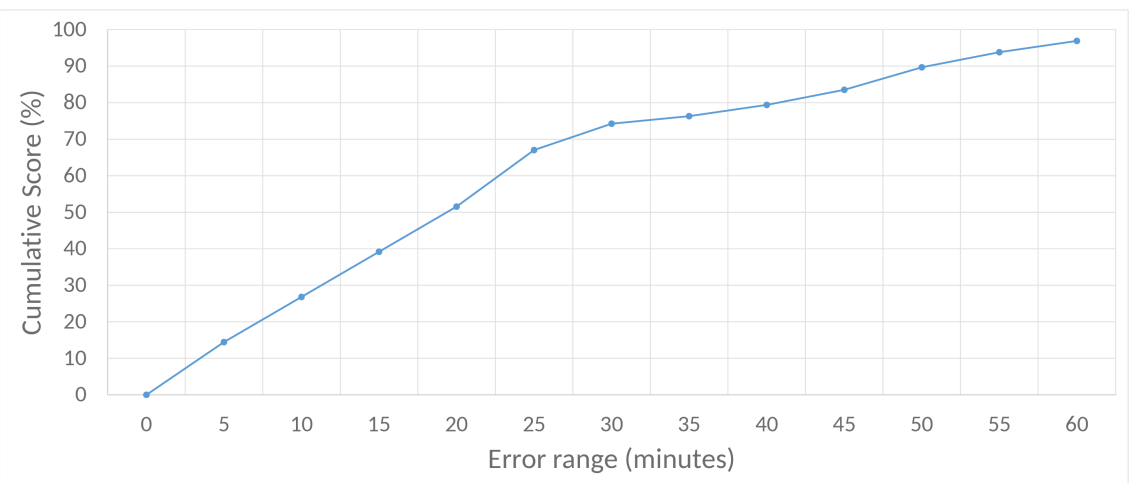

Fig. 7. The cumulative score of the sleep duration estimates at absolute error levels from 0 to 60 minutes.

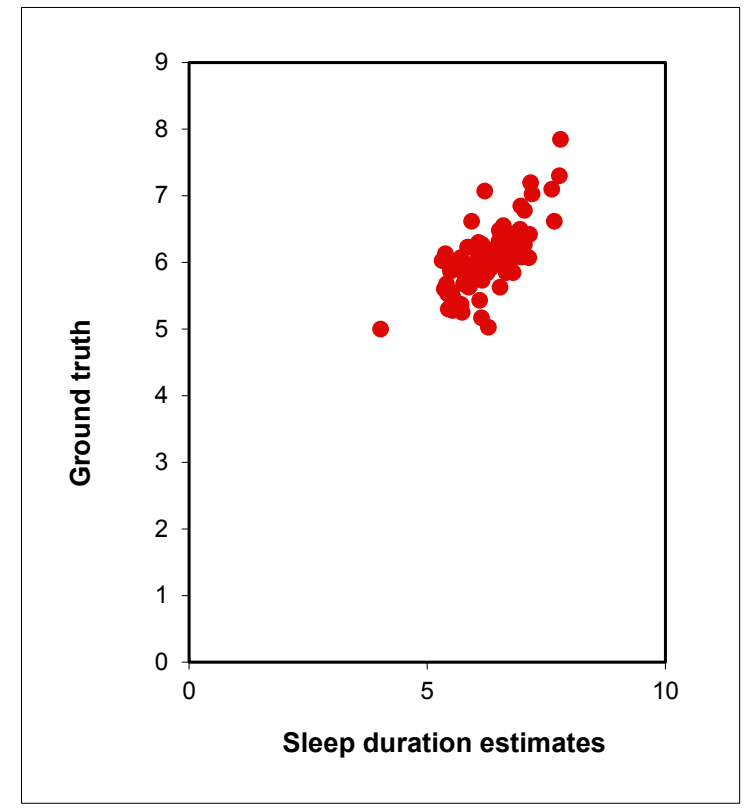

Fig. 8. The correlation coefficients between the sleep duration estimates and the ground truth. 
bility of sleep disorder. April (16), May (31) and December (35) have the least bathroom visits because the senior citizen was hospitalized. Finally, Figure 11 shows the probability of sleeping and visiting the bathroom in the middle of the night according to the preference of the senior citizen. The senior citizen prefers to sleep around the midnight with a probability of $50 \%$ and around $11 \mathrm{pm}$ with a probability of $30 \%$. He most likely visits the bathroom between 2 am to 5 am with probabilities of $27 \%, 29 \%$ and $29 \%$ respectively. There is a possibility of $15 \%$ that he goes to the bathroom around 1 am after he sleeps for one hour.

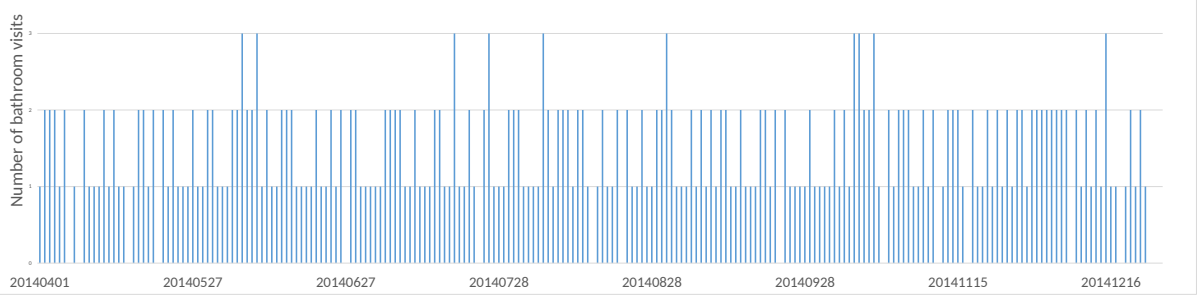

Fig. 9. The nightly bathroom visits per day from April to December.

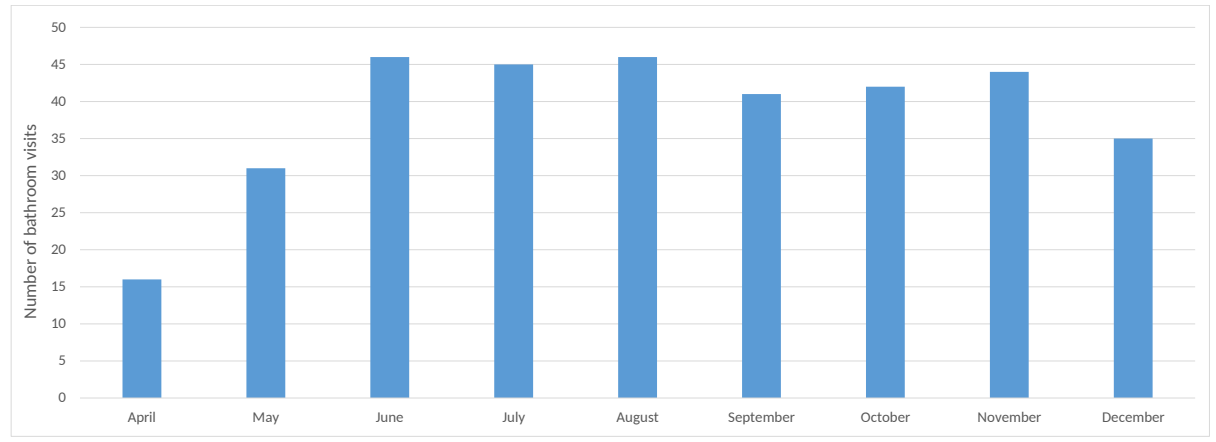

Fig. 10. The nightly bathroom visits per month from April to December.

\section{Conclusions}

In this paper, we presented a network of low-resolution visual sensors installed in a service flat of a senior citizen in Belgium. Our approach of detecting sleep 


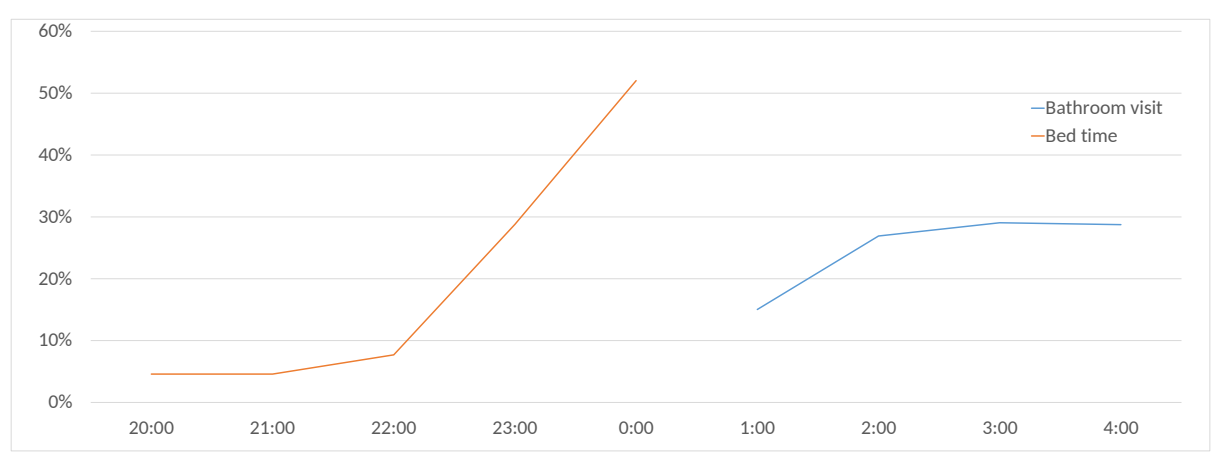

Fig. 11. The probability of sleeping and visiting the bathroom in the middle of the night.

durations is promising and does not require to install visual sensors inside the bedroom. We analyzed the sleep patterns and the nightly bathroom visits indirectly to indicate sleep disorders. The wake up time and sleep time were identified using a rule-based approach based on the motion patterns. The nightly bathroom visits were detected using SVM classifier. We collected 10 months of real-life video recordings. Our approach of estimating sleep durations against ground truth achieved MAE of 22.91 minutes and $r_{s}$ of 0.69 . Then, we studied the relation of the nightly bathroom visits and sleep disorder. The study showed several nights of sleep disorder. In the future, we aim at integrating and fusing visual information with data from other types of sensors (e.g. in the bed frame), and controllers (e.g. X10 or PIR) for detecting more activities to show health deterioration or improvement.

Acknowledgments This research has been performed in the context of the project "LittleSister" and the European AAL project "Sonopa". This research has been financed by the agency for Innovation by Science and Technology (IWT), the Belgian National Fund for Scientific Research (FWO Flanders), and iMinds.

\section{References}

1. Ancoli-Israel, S., Bliwise, D.L., Nrgaard, J.P.: The effect of nocturia on sleep. Sleep Medicine Reviews (2011)

2. Anliker, U., Ward, J., Lukowicz, P., Troster, G., Dolveck, F., Baer, M., Keita, F., Schenker, E., Catarsi, F., Coluccini, L., Belardinelli, A., Shklarski, D., Alon, M., Hirt, E., Schmid, R., Vuskovic, M.: Amon: a wearable multiparameter medical monitoring and alert system. Information Technology in Biomedicine, IEEE Transactions on 8(4), 415-427 (Dec 2004) 
3. Camilli, M., Kleihorst, R.: Demo: Mouse sensor networks, the smart camera. In: Distributed Smart Cameras (ICDSC), 2011 Fifth ACM/IEEE International Conference on. pp. 1-3 (Aug 2011)

4. Chen, Z., Lin, M., Chen, F., Lane, N., Cardone, G., Wang, R., Li, T., Chen, Y., Choudhury, T., Campbell, A.: Unobtrusive sleep monitoring using smartphones. In: Pervasive Computing Technologies for Healthcare (PervasiveHealth), 2013 7th International Conference on. pp. 145-152 (May 2013)

5. Doblhammer, G., Ziegler, U.: Future elderly living conditions in europe: Demographic insights. In: Backes, G.M., Lasch, V., Reimann, K. (eds.) Gender, Health and Ageing, pp. 267-292. VS Verlag fr Sozialwissenschaften (2006), http: //dx.doi.org/10.1007/978-3-531-90355-2_13

6. Eldib, M., Bo, N.B., Deboeverie, F., Nino, J., Guan, J., Van de Velde, S., Steendam, H., Aghajan, H., Philips, W.: A low resolution multi-camera system for person tracking. In: Image Processing (ICIP), 2014 IEEE International Conference on. pp. 378-382 (Oct 2014)

7. Foley, D., Ancoli-Israel, S., Britz, P., Walsh, J.: Sleep disturbances and chronic disease in older adults: Results of the 2003 national sleep foundation sleep in america survey. Journal of Psychosomatic Research 56(5), 497 - 502 (2004), http://www.sciencedirect.com/science/article/pii/S0022399904000327

8. Foley, D.J., Monjan, A.A., Brown, S.L., Simonsick, E.M., Wallace, R.B., Blazer, D.G.: Sleep complaints among elderly persons: an epidemiologic study of three communities. Pervasive Computing, IEEE 18(6), 425432 (Jan 1995)

9. Heinrich, A., Geng, D., Znamenskiy, D., Vink, J., de Haan, G.: Robust and sensitive video motion detection for sleep analysis. Biomedical and Health Informatics, IEEE Journal of 18(3), 790-798 (May 2014)

10. Hodgins, D., Bertsch, A., Post, N., Frischholz, M., Volckaerts, B., Spensley, J., Wasikiewicz, J.M., Higgins, H., Von Stetten, F., Kenney, L.: Healthy aims: Developing new medical implants and diagnostic equipment. Pervasive Computing, IEEE 7(1), 14-21 (Jan 2008)

11. Hoque, E., Dickerson, R.F., Stankovic, J.A.: Monitoring body positions and movements during sleep using wisps. In: Wireless Health 2010. pp. 44-53. WH '10, ACM, New York, NY, USA (2010), http://doi.acm.org/10.1145/1921081.1921088

12. Krger, B., Vgele, A., Herwartz, L., Terkatz, T., Weber, A., Garcia, C., Fietze, I., Penzel, T.: Sleep detection using a depth camera. In: Murgante, B., Misra, S., Rocha, A., Torre, C., Rocha, J., Falco, M., Taniar, D., Apduhan, B., Gervasi, O. (eds.) Computational Science and Its Applications ICCSA 2014, Lecture Notes in Computer Science, vol. 8579, pp. 824-835. Springer International Publishing (2014), http://dx.doi.org/10.1007/978-3-319-09144-0_57

13. Lee, J., Hong, M., Ryu, S.: Sleep monitoring system using kinect sensor. International Journal of Distributed Sensor Networks (2015)

14. Merilahti, J., Saarinen, A., Parkka, J., Antila, K., Mattila, E., Korhonen, I.: Longterm subjective and objective sleep analysis of total sleep time and sleep quality in real life settings. In: Engineering in Medicine and Biology Society, 2007. EMBS 2007. 29th Annual International Conference of the IEEE. pp. 5202-5205 (Aug 2007)

15. Metsis, V., Kosmopoulos, D., Athitsos, V., Makedon, F.: Non-invasive analysis of sleep patterns via multimodal sensor input. Personal and Ubiquitous Computing 18(1), 19-26 (2014), http://dx.doi.org/10.1007/s00779-012-0623-1

16. Muaremi, A., Bexheti, A., Gravenhorst, F., Arnrich, B., Troster, G.: Monitoring the impact of stress on the sleep patterns of pilgrims using wearable sensors. In: 
Biomedical and Health Informatics (BHI), 2014 IEEE-EMBS International Conference on. pp. 185-188 (June 2014)

17. Ni, H., Abdulrazak, B., Zhang, D., Wu, S., Yu, Z., Zhou, X., Wang, S.: Towards non-intrusive sleep pattern recognition in elder assistive environment. In: Yu, Z., Liscano, R., Chen, G., Zhang, D., Zhou, X. (eds.) Ubiquitous Intelligence and Computing, Lecture Notes in Computer Science, vol. 6406, pp. 96-109. Springer Berlin Heidelberg (2010), http://dx.doi.org/10.1007/978-3-642-16355-5_10

18. Nyan, B.B., Deboeverie, F., Eldib, M., Guan, J., Xie, X., Niño Castañeda, J., Van Haerenborgh, D., Slembrouck, M., Van de Velde, S., Steendam, H., Veelaert, P., Kleihorst, R., Aghajan, H., Philips, W.: Human mobility monitoring in very low resolution visual sensor network. Sensors 14(11), 20800-20824 (2014), http: $/ / \mathrm{dx}$.doi.org/10.3390/s141120800

19. Teixeira, T., Dublon, G., Savvides, A.: Physical and mental health effects of family caregiving. ENALAB technical report (2010)

20. Umlauf, M.G., Chasens, E.R., Greevy, R.A., Arnold, J., Burgio, K.L., Pillion, D.J.: Obstructive sleep apnea, nocturia and polyuria in older adults. Sleep 27(1), 139 $144(2004)$ 